\title{
HOT OFF THE 2021 ESH/ISH
} MEETING

\section{Introduction}

\author{
THOMAS UNGER \\ CARIM - School for Cardiovascular Research,Maastricht University, Maastricht, \\ The Netherlands.
}

DOI:10.30824/2106-2

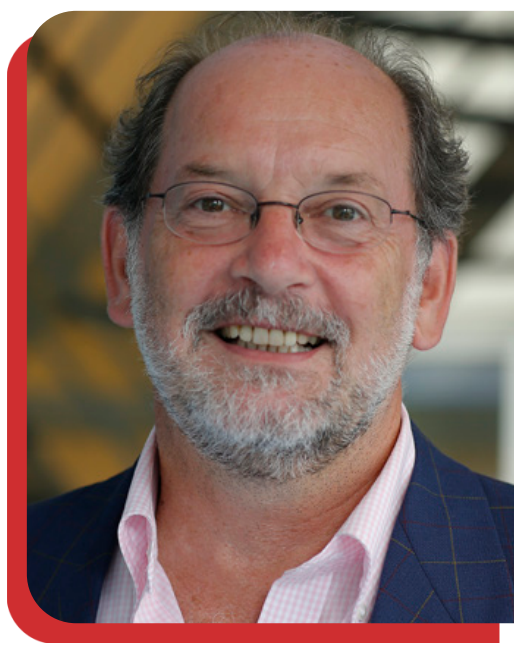

The Joint Meeting of the European Hypertension Society (ESH) and ISH together with the British and Irish Hypertension Society (BIHS) in Glasgow on April 11-14, 2021, was an overwhelming success and this despite the fact that it had to be performed exclusively on air due to the Corona Pandemic. Just a few figures to illustrate this: There were almost 5.400 registrations from 128 countries with almost 4.000 connections to the platform. In 118 sessions, 748 live speeches were held, and there were 127 hours of live transmission. An impressive organizational achievement demonstrating that a successful global hypertension congress can be held even under such adverse conditions. Great thanks to the congress president, Professor Anna Dominiczak, and to all those helping to realize this endeavor.

Content wise, the congress covered virtually all thinkable aspects of hypertension. For reasons of actuality with all the fresh impressions and memories, we at HTN News thought that the traditional section "Hot of the Press" in the journal should be devoted this time to some "hot" aspects presented at the Glasgow meeting. We invited some younger scientists to report briefly on their personal recollections of some topics which they thought were of high actuality and of general interest. This initiative granted us six short communication papers which are presented in the following.

Francisco Rios opens the roundelay with an article covering several presentations on immunity and hypertension, demonstrating the complexity of the immune system as a department not only of defense, but also of "internal welfare" and illustrating the tremendous impact of the various immune-regulatory aspects on cardiovascular disease including hypertension.

Thematically not far away, Nicolas F. Renna confronts us with a new medico-social-economic entity, called "Syndemic", which has been elicited by the Corona Virus 2 pandemic. He covers questions about a possible role of hypertension as a risk factor for the Covid-19 disease demonstrating, that (in agreement with statements of several hypertension societies) rather old age and comorbidities but not hypertension itself are the culprit. Likewise, antihypertensives such as ACE inhibitors and ARBs, which, on the basis of some mechanistic considerations, have also been accused to be risk factors for the Covid-19 disease, can be dismissed as risk factors as shown in recent clinical trials like BRACE CORONA or REPLACE COVID.

Hypertension in Pregnancy is the next topic, selected with respect to basics by Nayara Azinheira Nobrega da Cruz and Mariane Bertagnolli, and, relating to clinical aspects, by Bianca Davidson and Erika Jones. Basic issues discussed were the importance of impaired placental angiogenesis in preeclampsia, the role of mesenchymal stem cells and various aspects of biomarkers such as sFit-1. The clinical paper covers in detail the so-called CALIBER study from the UK characterizing hypertension during pregnancy as an independent cardiovascular risk factor and stressing the fact that women with hypertension during pregnancy ought to be monitored closely after delivery as recommended by the recent ISH Hypertension Guidelines. 
Finally the salt issue. Still a matter of controversy after so many years of research, discussions and official recommendations. Basic aspects are dealt with by Cesar Romero introducing us to a new regulatory entity called "natriuretic-ureotelic regulation". Another topic he discusses is the role of chloride vs other anions as partners of sodium. In experiments on genetically hypertensive rats, $\mathrm{F}$. Luft and al (Clin Sci 1988;74(6):577-85) have already demonstrated many years ago that drinking a sodium chloride- but not sodium bicarbonate solution can increase blood pressure. New basic data discussed at the Glasgow meeting may shed some light on underlying mechanisms. In addition, exciting new results point to an association of high salt intake and cognitive impairment involving the Tau protein which has long been recognized to be involved in Alzheimer's disease. The last paper of this section by Neusa Jessen deals in a very balanced way with several clinical aspects of the salt story discussed at the Glasgow meeting. New data by Franz Messerli and colleagues pointing to a lack of an association between salt intake and premature death are discussed in the light of the large body of evidence accusing a high salt diet to be an important risk factor for hypertension and cardiovascular disease. Recent data suggest that some biomarkers may be able to better distinguish between salt-sensitive and salt-resistant individuals, and there was even a recommendation that salt food "fortification" with nitrate-rich vegetable extracts may be a better solution to the problem than reducing salt in the diet.

\title{
Immunity and Inflammation in Hypertension - A Long Way To Go
}

\author{
FRANCISCO J RIOS \\ Institute of Cardiovascular and Medical Sciences, BHF Glasgow Cardiovascular Research \\ Centre, University of Glasgow, \\ Glasgow, UK.
}

\section{DOI:10.30824/2106-3}

The immune system is often defined as a complex network to protect the body against the invasion of microorganisms. In fact, what we most know about the immune response is derived from studies observing the immune response over a challenge mediated by pathogens or non-self-molecules. This traditional concept is not too far from the truth; however, it is a rather simplistic way to define such a complex system. According to Prof Cohen "The immune system has earned a reputation, justly, for its role as protector of the body against foreign invaders. However, the immune system is not only a department of defence, it also functions as a department of internal welfare" ${ }^{\prime \prime}$. Following this line of thinking, we should dare to consider that the main function of the immune system is to control the body homeostasis and, in only extreme cases this system faces an infection. This complex network is achieved by a group of circulating and tissue resident cells that patrol vessels and tissues to scavenge cells debris or DAMPS (Danger Associated Molecular
Patterns) to avoid unnecessary activation. In stress conditions, the immune system acts to eliminate the stressor agent and ultimately to return the system to the homeostatic state ${ }^{2}$. However, as the old proverb says: "the road to hell is paved with good intentions"; Situations where stressor agents are constantly produced will lead to uncontrolled immune/inflammatory activation, and a response that was primarily induced to eliminate the stressor agent, now can potentially lead to chronic inflammatory diseases and tissue damage.

Given the impact of the immune system in cardiovascular diseases and that patients with high blood pressure exhibit increased plasma inflammatory mediators and immune cells infiltration in the cardiovascular and renal tissues, it comes as no surprise that biomedical research is focused on applying these observations to hypertension research, and this has been under intense investigation in the past 15 years ${ }^{3,4,5}$. 